\title{
Nemesis, Tyche, Planet Nine Hypotheses. I. Can We Detect the Bodies Using Gravitational Lensing?
}

\author{
J. P. Philippov ${ }^{1,3}$ and M. I. Chobanu ${ }^{2}$ \\ ${ }^{1}$ Samara State University, Academica Pavlova st 1, 443011, Samara, Russian Federation \\ ${ }^{2}$ Samara International Aerospace Lyceum, Lukacheva st 45, 443086, Samara, Russian Federation \\ ${ }^{3}$ Email: yuphil@mail.ru
}

(Received December 21, 2015; Accepted July 18, 2016)

\begin{abstract}
In this paper, the hypothesis of the existence of a massive dark body (Nemesis, Tyche, Planet Nine, or any other transPlutonian planet) at the Solar system periphery is analysed. Basic physical properties and orbital characteristics of such massive bodies are considered. The problem of the definition of a scattering angle of a photon in the gravitational field of a spherical lens is studied. It is shown that, the required value of the scattering angle can be measured for the cases of Nemesis and Tyche. The formation of gravitational lensing images is studied here for a point mass event. It is demonstrated that in most cases of the close rapprochement of a source and the lens (for Nemesis and Tyche), it is possible to resolve two images. The possibility of resolving these images is one of the main arguments favouring the gravitational lensing method as its efficiency in searching for dark massive objects at the edge of the Solar System is higher than the one corresponding to other methods such as stellar occultation. For the cases of Planet Nine and any other trans-Plutonian planet, the strong gravitational lensing is impossible because at least one of the images is always eclipsed.
\end{abstract}

Keywords: planets and satellites: detection - planets and satellites: general - planets and satellites: Oort Cloud - stars: brown dwarfs - gravitational lensing: strong - gravitational lensing: scattering

\section{INTRODUCTION}

At the beginning of the 21 st century and given the knowledge acquired during the 20th century as a result of the investigations of stars in the main sequence of the H-R diagram has become clear that approximately a half of the stars in our galaxy belong to binary or multiple star systems (Duquennoy \& Mayor 1991; Kononovich \& Moroz 2004). Taking this observational fact into account, the following questions arise: Is the Sun the only star in the Solar System? Does it have a companion?

A number of recent observational facts have provided a preliminary answer to the questions posed above almost discarding the existence of a stellar or substellar companion to the Sun. Data from the WISE infrared space telescope (Luhman 2014) showed no evidence of a planet the size of Saturn out to $28000 \mathrm{AU}$, Jupiter out to $82000 \mathrm{AU}$, or a Jupiter-sized brown dwarf out to $26000 \mathrm{AU}$ at the locations suggested by Matese \& Whitmire (1986). The version of the Nemesis hypothesis (Davis, Hut, \& Muller 1984) assuming that the companion of the Sun was a common M-dwarf star has been ruled out by full sky surveys, the latest of which is the NASA WISE mid-IR survey (Luhman 2014). However, the alternative version of the Nemesis hypothesis (Whitmire \& Jackson 1984) assuming that the Sun's companion was a substellar object with a mass between 0.07 and 0.0002 solar masses may still be viable. The lower end of this mass range is not ruled out by WISE or any other full sky survey given the fact that the object would likely be closer to aphelion today (Whitmire 2016). Moreover, there is still room for the existence of trans-Plutonian planets even ten times as massive as the Earth at a distance of more than $195 \mathrm{AU}$ from the Sun (Trujillo \& Sheppard 2014; de la Fuente Marcos \& de la Fuente Marcos 2014; de la Fuente Marcos, de la Fuente Marcos, \& Aarseth 2015; Batygin \& Brown 2016).

Now that the existence of another stellar, substellar,or Jovian-like planetary body in the Solar System is very unlikely, if other massive bodies exist they must be planetary in nature and no more massive than about ten Earth masses being located at a distance of more than $150 \mathrm{AU}$ from the Sun. These putative cold and dark objects located at large distances from the Sun are difficult to detect even in the infrared region of the electromagnetic spectrum. In some cases, they could have probably been detected by past surveys 
(Batygin \& Brown 2016; Linder \& Mordasini 2016). These dark bodies moving very slowly at the Solar System periphery have huge orbital periods and the chances to detect them using conventional techniques are remote. Because of this, the study of gravitational perturbations and stellar occultations produced by such massive objects, and the analysis of their effects on the light coming from distant stars have become crucial methods to be used in their detection (Roques, Moncuquet, \& Sicardy 1987; Gaudi \& Sackett 2000; Cooray 2002; Gaudi \& Bloom 2005; Bickerton, Kavelaars, \& Welch 2008; Heyl 2010; Zhdanov \& Gorpinchenko 2012).

The possibility of detecting a far away and dark massive body indirectly by means of its effects on the light coming from distant stars could be the basis of a new approach to the search for a trans-Plutonian planet at the Solar System periphery by using the gravitational lensing method (GLM). The GLM was first mentioned by the Russian physicist O. Chwolson (Chwolson 1924) and then studied quantitatively by A. Einstein (Einstein 1936). It is based on the effect of gravitational lensing consisting in bending of light beams coming from distant stars by a dark and massive point-like object (lens). Two or more images of a source are formed by the lens. If these separate images are resolved by the telescope, then it is called strong lensing. Otherwise, it is called gravitational microlensing and in this case, the gravitational field of the dark and massive point-like object affects starlight resulting in a great increase of the brightness of the stars. The gravitational microlensing effect is the basis of the gravitational microlensing method (GMM) which has been first proposed by B. Paczynski (Paczynski 1986a, 1986b). The realisation of the method in practice has taken place at the beginning of the 21st century (Zhu et al. 2015) and in the near future, it may be used to search for a putative dark massive planet at the edge of the Solar System.

The main purpose of this work is to determine the basic physical properties of a massive dark body (this can be a brown dwarf-Nemesis, a giant planet as massive or more massive than Jupiter-Tyche, or a trans-Plutonian planet as massive or more massive than Earth but less massive than Neptune-Planet Nine (P9) or any other trans-Plutonian planet) which may exist at the Solar System periphery and to perform a quantitative analysis of the possibility of detection of such a body using the GLM.

\section{ABOUT THE FACTS SUPPORTING THE EXISTENCE OF TRANS-PLUTONIAN PLANETS}

In this section, we present the most important facts supporting indirectly the hypothesis of the existence of a massive dark planet at the Solar System periphery.

I. As explained above, approximately $50 \%$ of all main sequence stars are part of double or multiple stars systems (Duquennoy \& Mayor 1991; Kononovich \& Moroz 2004). Based on this observational fact, it had been suggested that

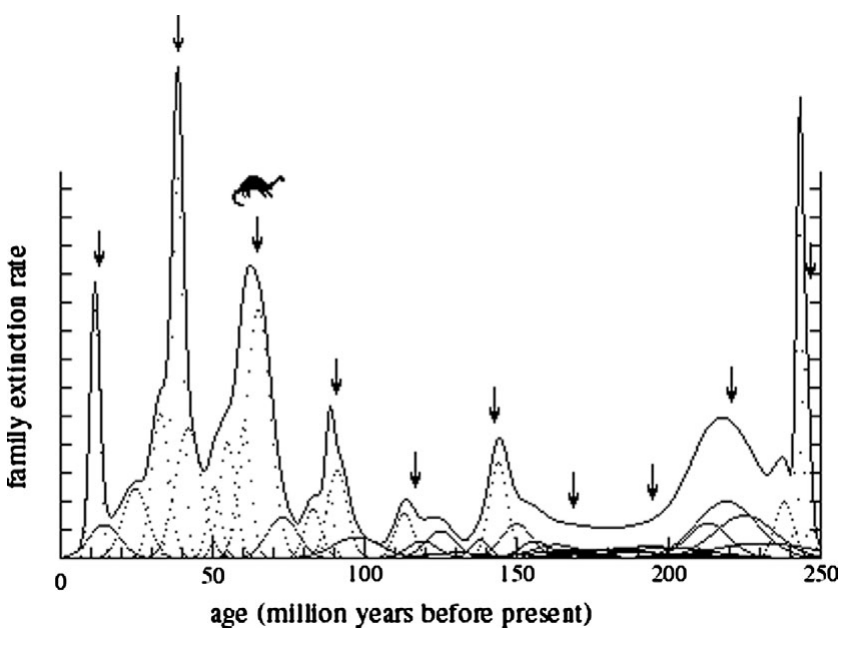

Figure 1. Mortality curve of living organisms on Earth, according to Raup \& Sepkoski (1984).

the Sun may have a gravitationally bound companion star, i.e., the Sun could be part of a binary system. However, recent WISE space telescope observational findings (Luhman 2014) have decreased the credibility of this hypothesis to a minimum level. Data from the WISE infrared space telescope (Luhman 2014) showed no evidence of a Jupiter-sized brown dwarf out to $26000 \mathrm{AU}$ at the locations suggested by Matese \& Whitmire (1986). Only the lower end of the mass range proposed in Whitmire and Jackson's Nemesis hypothesis (Whitmire \& Jackson 1984), i.e., a brown dwarf companion to the Sun with a mass of 0.0002 solar masses, has not yet been ruled out by NASA WISE or any other full sky survey (Whitmire 2016).

II. Palaeontologists J. Sepkoski and D. M. Raup (Raup \& Sepkoski 1984) published the results of the analysis of geological layers, corresponding to the different eras, spanning the last 250 Myr. They found 12 events corresponding to sharp increases in mortality of living organisms on Earth. The time interval between two of these events is about 27 Myr (see Figure 1). Raup and Sepkoski concluded that such events (including the alleged disappearance of the dinosaurs $65 \mathrm{Myr}$ ago) happened regularly and could have an extraterrestrial origin, in particular, a gravitational disturbance in the Oort cloud of comets, caused by the presence of a massive dark body in the outskirts of the Solar System. Gravitational perturbations generated by such a dark body may have led to a cometary migration to the inner Solar System and some of these perturbed comets, or a near-Earth asteroid whose orbit was distorted by their presence, could have collided with Earth. A putative asteroidal or cometary impact could have caused the global catastrophe that led to the demise of a great amount of living organisms on Earth. Recently, the topic of the periodic mass extinctions and the Planet $X$ hypothesis has been revisited (Whitmire 2016). D. P. Whitmire concludes that the Planet X hypothesis can explain the $27 \mathrm{Myr}$ periodicity in the fossil extinction record. In this case, the period in 
question is associated with the perihelion precession of the inclined orbit of a trans-Neptunian planet (more massive than Earth but less massive than Neptune). The existence of such a trans-Neptunian super-Earth planet has been proposed to explain the observational fact that inner Oort cloud objects such as Sedna and 2012 VP113 have perihelia that lie near the ecliptic plane (Trujillo \& Sheppard 2014; de la Fuente Marcos \& de la Fuente Marcos 2014; Iorio 2014; de la Fuente Marcos et al. 2015; Batygin \& Brown 2016).

III. Long-term studies of comets have shown that new comets, coming to us from the Solar System periphery, appear regularly: In some years, the number of comets is significantly higher than in others. Most of the comets are approaching to the Earth at perihelia coming from the Oort cloud (Yarris 1987). The periodicity of such comets appears to be directly related to the periodicity in movement of an unknown massive celestial body that may exist in the outskirts of the Solar System.

IV. Pluto, Eris, Sedna, and other trans-Neptunian and extreme trans-Neptunian objects (ETNOs) have very elongated orbits and large values of orbit inclinations to the plane of the ecliptic. In the case of Sedna, its extremely elliptical orbit as well as its dynamical evolution cannot be convincingly explained by the current model of the Solar System that includes only eight major planets revolving around the Sun (Brown 2004). However, these extremely elongated orbits and high inclinations can be easily explained by the existence of a massive celestial body located at the Solar System periphery. From such a vantage location, a putative massive dark planet may induce gravitational perturbations on the trans-Neptunian objects (Gomes, Matese, \& Lissauer 2006).

V. The study of the circumstellar discs of the stars HD53143 and HD139664 with HST (Hubble space telescope) has shown that both discs appear to be in a stable state of equilibrium (Kalas et al. 2006). The dust disk of each star is divided into two zones. One of them is a wide belt with a gradually decreasing density of matter, starting with $r \geq$ $50 \mathrm{AU}$ from the star. The second disc is located in the range of 20-30 AU from the star and has a distinct outer edge, the density of material in the disk falls sharply here. This may indicate the presence of a companion star or planet. The object's orbit runs along the outer edge of the disc. It constantly cuts the edge of the circumstellar disc, protecting it from erosion. Similar effects are observed in the rings of Saturn. The existence of these circumstellar discs, even considering that they may include belts similar to the Kuiper belt, has some implications for the case of the Solar System, despite of the physical properties of the stars HD53143 and HD139664 are slightly different than the ones corresponding to the Sun. Recently, S. J. Kenyon and B. C. Bromley have shown (Kenyon \& Bromley 2015, 2016) how it is possible-for mechanisms acting on a stellar protoplanetary disc - to form planets as massive or even 15 times as massive as the Earth at distances within the range 125-750 AU from a solar-type star. Moreover, observational inference of extrasolar planetary systems such as HR 8799 (Marois et al. 2008) suggests that planets can indeed occupy exceptionally wide orbits.

VI. The results of Monte Carlo simulations (de la Fuente Marcos \& de la Fuente Marcos 2014) and numerical integrations using an N-body model applied to comet 96P/Machholz 1 (de la Fuente Marcos et al. 2015) strongly suggest that trans-Plutonian planets may exist. According to these authors, beyond the orbit of Pluto at distances of about 205264, 346-416, 610-692, and 972-1001 AU from the Sun-a number of massive planets may be located.

VII. According to new ALMA (Atacama Large Millimeter/submillimeter Array) observational findings (Liseau et al. 2015; Vlemmings et al. 2015), two new objects of the Solar System have been discovered. One of them is a fast moving single object (with proper motion 87 arcsec $\mathrm{yr}^{-1}$ ), named Gna, that could be a large Centaur with a size within the range $220-880 \mathrm{~km}$ at a distance of $12-20 \mathrm{AU}$ if it is gravitationally bound or a low-mass brown dwarf beyond $4000 \mathrm{AU}$ if it is not gravitationally bound (Vlemmings et al. 2015). The nature of this object is not yet clear, its geocentric distance is unknown. The other object shares the high proper motion of alpha Centauri and has a prograde motion (Liseau et al. 2015). According to Liseau et al., the object could be a super-Earth (with a radius one and a half times that of the Earth and located at a distance of about 300 AU from the Sun) or a super-cool brown dwarf (at a distance of about $20000 \mathrm{AU})$. Considering that WISE space telescope infrared observations found no evidence of a planet the size of Saturn out to $28000 \mathrm{AU}$, Jupiter out to $82000 \mathrm{AU}$, or a Jupiter-sized brown dwarf out to $26000 \mathrm{AU}$ at the locations suggested by Matese \& Whitmire (Luhman 2014), the first option, i.e., the existence of a trans-Plutonian planet 2-15 times as massive as the Earth at a distance of approximately $300 \mathrm{AU}$ from the Sun, is the most probable one. However, it must be emphasised here that both discoveries by ALMA are no more than unconfirmed tentative candidates to be members of the Solar System. They may well correspond to false or spurious detections until proven otherwise. Clearly, more observations of these objects are needed to confirm their existence.

VIII. Very recently, K. Batygin and M. E. Brown (Batygin \& Brown 2016; Brown \& Batygin 2016) have found that the observed orbital alignment of the ETNOs can be maintained by a distant, eccentric trans-Plutonian planet with a mass close to that of Neptune or lower lying approximately in the same orbital plane defined by some of the ETNOs, i.e., with inclination of approximately $30^{\circ}$, and with perihelion $180^{\circ}$ away from the perihelia of the ETNOs. In addition to accounting for the observed orbital alignment, the existence of such a planet naturally explains the presence of highperihelion Sedna-like objects, as well as the known collection of high semi-major axis objects with inclinations between $60^{\circ}$ and $150^{\circ}$ whose origin was previously unclear. However, although this hypothesis has been successful in generating a distant population of small bodies whose orbits exhibit alignment in physical space, there are observational aspects of the distant Kuiper Belt that the P9 hypothesis is unable 
Table 1. Basic properties of Nemesis and its orbit.

\begin{tabular}{|c|c|c|c|}
\hline Property & Range & & Reference \\
\hline Mass $\left(\mathfrak{M}_{\mathrm{N}}\right)$ & $\begin{array}{c}\mathfrak{M}_{\mathrm{N}} \leq 0.042 \mathrm{M}_{\odot}, \\
2.00 \times 10^{-4} \leq \mathfrak{M}_{\mathrm{N}} \leq 7.00 \times 10^{-2} \mathrm{M}_{\odot} \\
1.241 \times 10^{-2} \leq \mathfrak{M}_{\mathrm{N}} \leq 7.00 \times 10^{-2} \mathrm{M}_{\odot}\end{array}$ & (1) & $\begin{array}{l}\text { Bhalerao \& Vahia (2005) } \\
\text { Whitmire \& Jackson (1984) } \\
\text { This work }\end{array}$ \\
\hline $\operatorname{Radius}\left(\mathfrak{R}_{\mathrm{N}}\right)$ & $\mathfrak{R}_{\mathrm{N}} \approx 71492 \mathrm{~km}$ & (2) & This work \\
\hline Eff.temperature $\left(T_{\text {eff }}^{(\mathrm{N})}\right)$ & $3 \times 10^{2} \leq T_{\mathrm{eff}}^{(\mathrm{N})} \leq 3 \times 10^{3} \mathrm{~K}$ & (3) & This work \\
\hline Semi-major axis $\left(a_{\mathrm{N}}\right)$, eccentricity $\left(\varepsilon_{\mathrm{N}}\right)$ & $a_{\mathrm{N}}=8.8 \times 10^{4} \mathrm{AU}, \quad \varepsilon_{\mathrm{N}}=0.9$ & (4) & Whitmire \& Jackson (1984), This work \\
\hline Orbital period $\left(T_{\mathrm{N}}\right)$ & $\begin{array}{c}10^{4} \leq T_{\mathrm{N}} \leq 6.2 \times 10^{7} \mathrm{yr} \\
T_{\mathrm{N}}=2.6 \times 10^{7} \mathrm{yr}\end{array}$ & & $\begin{array}{l}\text { Bhalerao \& Vahia (2005) } \\
\text { Whitmire \& Jackson (1984), This work }\end{array}$ \\
\hline Heliocentric distance $\left(r_{\mathrm{N}}\right)$ & $8.8 \times 10^{3} \leq r_{\mathrm{N}} \leq 1.672 \times 10^{5} \mathrm{AU}$ & $(5)$ & This work \\
\hline
\end{tabular}

to address. Specifically, the apparent clustering of arguments of perihelia near argument of perihelion zero in the 150-250 AU region remains somewhat puzzling (Batygin \& Brown 2016). To explain such a feature, another massive perturber, perhaps with mass within the range 2-15 Earth masses, at a distance of $264 \mathrm{AU}$ from the Sun in a moderately eccentric orbit with low inclination could be required (de la Fuente Marcos et al. 2015).

On the other hand, to explain the unusual orbital distribution of distant Kuiper belt objects (KBOs) or ETNOs, Fienga et al. (2016) have advocated the existence of a super-Earth planet in the outer Solar System, based on the P9 hypothesis proposed by Batygin \& Brown (2016). Making use of the most sensitive data set, the Cassini radio ranging data and the INPOP planetary ephemerides model (Fienga et al. 2008, 2009, 2011), Fienga et al. (2016) have shown that a transPlutonian planet with mass 10 Earth masses, moving along an orbit with a semi major axis of $700 \mathrm{AU}$ and an eccentricity of 0.6 , may exist if its true anomaly does not belong to the interval $\left[-130^{\circ},-100^{\circ}\right]$ and $\left[-65^{\circ}, 85^{\circ}\right]$. Moreover, Fienga et al. (2016) point out the most probable position of P9 given by a true anomaly $117.8^{\circ}{ }_{-10^{\circ}}^{\circ}$. Using Cassini data up to the epoch 2014.4 and the extrapolated Cassini data up to 2020, Fienga et al. (2016) were able to identify new and more stringent restrictions on the range of possible values for the true anomaly. If the Cassini mission is extended until 2020 , it would thus allow to state for the non-existence of P9 on the intervals of true anomaly given by $\left[-132^{\circ}, 106.5^{\circ}\right]$. Therefore, the corresponding range of available heliocentric distances for $\mathrm{P} 9$ is constrained by Cassini data to 549-1120 AU, being $q=280 \mathrm{AU}$ the perihelion distance of $\mathrm{P} 9$.

\section{PHYSICAL PROPERTIES OF NEMESIS, TYCHE, PLANET NINE, AND ANY OTHER TRANS-PLUTONIAN PLANET}

Whitmire \& Jackson (1984) and Davis et al. (1984) first proposed the hypothesis of the existence of a stellar companion to the Sun which moves with it around a common centre of mass and together form a binary star (see e.g., Muller
2002). It was named Nemesis ${ }^{1}$. Since then, other scientists have supported the hypothesis of Whitmire, Jackson, and Davis, and they have accomplished a theoretical analysis of the properties of the object (Bhalerao \& Vahia 2005). However, after the publication of the results obtained by the WISE space telescope (Luhman, 2014) that found no evidence of a Jupiter-sized brown dwarf out to $26000 \mathrm{AU}$ at the locations suggested by Matese \& Whitmire (1986), the chances for the existence of Nemesis are very slim. However, a brown dwarf companion to the Sun with a mass of 0.0002 solar masses, which corresponds to the lower end of the mass range proposed by Whitmire and Jackson (Whitmire \& Jackson 1984), has not yet been ruled out by NASA WISE or any other full sky survey (Whitmire 2016).

Table 1 shows a summary of numerical values of basic characteristics of Nemesis and its orbit that have been used in this research. We propose a permissible range of mass values (which is expressed in solar masses, $\mathrm{M}_{\odot}=1.989 \times 10^{30}$ $\mathrm{kg}$ ) and the effective temperature of Nemesis' surface corresponding to a brown dwarf, according to Burrows et al. (1993). We also take into account that a typical brown dwarf has a radius approximately equal to Jupiter's radius $\Re_{\mathrm{N}} \approx R_{\mathrm{J}}{ }^{2}$

Matese et al. (2011) proposed the hypothesis of the existence of a massive cosmic body, a gas giant planet, in the Oort cloud. It was named Tyche ${ }^{3}$. Data analysis of long-term observations of the orbits of comets approaching the Sun led the research team to the conclusion that about $20 \%$ of the comets falling into the inner part of the Solar system are attracted by the gravitational force of a massive cosmic object in the Oort cloud. According to these authors, the massive object would have a mass at least 1.4 times higher than Jupiter's mass. But if it was a star, the percentage of comets, captured by the gravitational field, would be much higher. However, after the publication of the results obtained by the WISE

\footnotetext{
${ }^{1}$ Nemesis was named after the ancient Greek goddess of revenge.

${ }^{2}$ Hereinafter $R_{\mathrm{J}}=71492 \mathrm{~km}$ is the radius of Jupiter, $M_{\mathrm{J}}=1.899 \times 10^{27} \mathrm{~kg}$ is its mass.

${ }^{3}$ Tyche was named after the goddess of fortune, Nemesis' sister. This was done to highlight that Tyche is a planet, not a star.
} 
Table 2. Basic properties of Tyche and its orbit.

\begin{tabular}{|c|c|c|c|}
\hline Property & Range & & Reference \\
\hline \multirow[t]{2}{*}{ Mass $\left(\mathfrak{M}_{\mathrm{T}}\right)$} & $1.4 \leq \mathfrak{M}_{\mathrm{T}} \leq 4 M_{\mathrm{J}}$ & & Matese, Whitmire, \& Lissauer (2006); \\
\hline & $1 \leq \mathfrak{M}_{\mathrm{T}} \leq 13 M_{\mathrm{J}}$ & (6) & This work \\
\hline $\operatorname{Radius}\left(\Re_{\mathrm{T}}\right)$ & $\mathfrak{R}_{\mathrm{T}} \approx 71492 \mathrm{~km}$ & (7) & This work \\
\hline Eff. temperature $\left(T_{\mathrm{eff}}^{\mathrm{T}}\right)$ & $T_{\mathrm{eff}}^{\mathrm{T}} \approx 200 \mathrm{~K}$ & (8) & Matese et al. (2011) \\
\hline Heliocentric distance $\left(r_{\mathrm{T}}\right)$ & $2.0 \times 10^{3} \mathrm{AU} \leq r_{\mathrm{T}} \leq 1.20 \times 10^{5} \mathrm{AU}$ & (9) & This work \\
\hline Orbital period $\left(T_{\mathrm{T}}\right)$ & $\begin{array}{c}T_{\mathrm{T}}=1.8 \times 10^{6} \mathrm{yr} \\
8.9 \times 10^{4} \leq T_{\mathrm{T}} \leq 4.16 \times 10^{7} \mathrm{yr}\end{array}$ & (10) & $\begin{array}{l}\text { Matese et al. (2011) } \\
\text { This work }\end{array}$ \\
\hline
\end{tabular}

Table 3. Basic properties of Planet Nine (P9) and its orbit.

\begin{tabular}{|c|c|c|c|}
\hline Property & Range & & Reference \\
\hline Mass $\left(\mathfrak{M}_{\mathrm{p} 9}\right)$ & $\begin{array}{r}10 M_{\oplus} \leq \mathfrak{M}_{\mathrm{P} 9} \leq 15 M_{\oplus} \\
5 M_{\oplus} \leq \mathfrak{M}_{\mathrm{P} 9} \leq 20 M_{\oplus} \\
5 M_{\oplus} \leq \mathfrak{M}_{\mathrm{P} 9} \leq 50 M_{\oplus}\end{array}$ & (11) & $\begin{array}{l}\text { Batygin \& Brown (2016), This work } \\
\text { Brown \& Batygin (2016) } \\
\text { Fortney et al. (2016) }\end{array}$ \\
\hline Radius $\left(\Re_{\mathrm{P} 9}\right)$ & $\begin{array}{l}2 R_{\oplus} \leq \mathfrak{R}_{\mathrm{P} 9} \leq 4 R_{\oplus} \\
2.9 R_{\oplus} \leq \mathfrak{R}_{\mathrm{P} 9} \leq 4.4 R_{\oplus} \\
2.9 R_{\oplus} \leq \mathfrak{M}_{\mathrm{P} 9} \leq 8.3 R_{\oplus}\end{array}$ & (12) & $\begin{array}{l}\text { Batygin \& Brown (2016), This work } \\
\text { Brown \& Batygin (2016) } \\
\text { Fortney et al. (2016) }\end{array}$ \\
\hline $\begin{array}{l}\text { Semi-major axis }\left(a_{\mathrm{P} 9}\right) \text {, eccentricity }\left(\varepsilon_{\mathrm{P} 9}\right) \\
\text { perihelion }\left(q_{\mathrm{P} 9}\right)\end{array}$ & $\begin{array}{r}a_{\mathrm{P} 9}=7 \times 10^{2} \mathrm{AU}, \quad \varepsilon_{\mathrm{P} 9}=0.6 \\
380 \mathrm{AU} \leq a_{\mathrm{P} 9} \leq 980 \mathrm{AU} \\
150 \mathrm{AU} \leq q_{\mathrm{P} 9} \leq 350 \mathrm{AU}\end{array}$ & (13) & $\begin{array}{l}\text { Batygin \& Brown (2016), This work } \\
\text { Brown \& Batygin (2016) }\end{array}$ \\
\hline Heliocentric distance $\left(r_{\mathrm{P} 9}\right)$ & $\begin{aligned} 280 \mathrm{AU} & \leq r_{\mathrm{P} 9} \leq 1120 \mathrm{AU} \\
549 \mathrm{AU} & \leq r_{\mathrm{P} 9} \leq 1120 \mathrm{AU} \\
549 \mathrm{AU} & \leq r_{\mathrm{P} 9} \leq 1718 \mathrm{AU}\end{aligned}$ & (14) & $\begin{array}{l}\text { Batygin \& Brown (2016), This work } \\
\text { Fienga et al. (2016) } \\
\text { Brown \& Batygin (2016), Fienga et al. } \\
\quad(2016)\end{array}$ \\
\hline Orbital period $\left(T_{\mathrm{P} 9}\right)$ & $\begin{array}{l}T_{\mathrm{P} 9}=18520 \mathrm{yr} \\
7408 \mathrm{yr} \leq T_{\mathrm{P} 9} \leq 30679 \mathrm{yr}\end{array}$ & (15) & $\begin{array}{l}\text { Batygin \& Brown (2016), This work } \\
\text { Brown \& Batygin (2016) }\end{array}$ \\
\hline
\end{tabular}

space telescope (Luhman, 2014) that found no evidence of a Jupiter-sized brown dwarf out to $26000 \mathrm{AU}$ at the locations suggested by Matese \& Whitmire (1986), the possibility of the existence of Tyche is more remote than ever.

Table 2 shows a summary of numerical values of Tyche's basic physical properties. As for the range of possible values for Tyche's mass (expressed in Jupiter masses $-M_{\mathrm{J}}$ ), a range of values allowed for planets like Jupiter (Jovian planets) is used (Burrows et al. 1993). We have taken into account that the orbit of Tyche should not go beyond the solar Hill sphere (which external radius is at least $1.20 \times 10^{5} \mathrm{AU}$ ) to determine the allowable heliocentric distances (see inequality (9) in Table 2). The lower limit for the semi-major axis of the orbit of Tyche is determined by the internal radius of the Oort cloud, based on the assumption that the orbit of Tyche is fully included in the Oort cloud.

Trujillo \& Sheppard (2014) first proposed the existence of a trans-Plutonian planet to explain the orbital behaviour of the distant KBO 2012 VP113. They compared the orbit of 2012 VP113 with that of the dwarf planet Sedna and con- cluded that the orbits of these objects suggest that a massive object, a planet heavier than Earth, could exist at around 250 AU moving along an almost circular orbit. An alternative scenario, the P9 hypothesis, has been proposed by Batygin \& Brown (2016). These authors argue that the gravitational perturbation generated by a massive outer planet moving along an eccentric and moderately inclined orbit with semi-major axis of $700 \mathrm{AU}$ (P9) would be the most likely explanation for the perihelia clustering of the orbits of six distant KBOs.

Tables 3 and 4 show a summary of numerical values of the basic physical properties of P9 and any other trans-Plutonian planet with different but plausible values. The majority of the parameter ranges have been taken from Batygin \& Brown (2016). For comparison, we have also provided the ranges in mass, radius, perihelion distance, semi-major axis, and heliocentric distance for P9 according to Brown \& Batygin's more recent work (Brown \& Batygin 2016). We have also taken into account the results in de la Fuente Marcos \& de la Fuente Marcos (2014), Iorio (2014), Trujillo \& Sheppard (2014), de la Fuente Marcos et al. (2015), Liseau et al. (2015), 
Table 4. Basic properties of a trans-Plutonian planet (TP), other than P9, and its orbit.

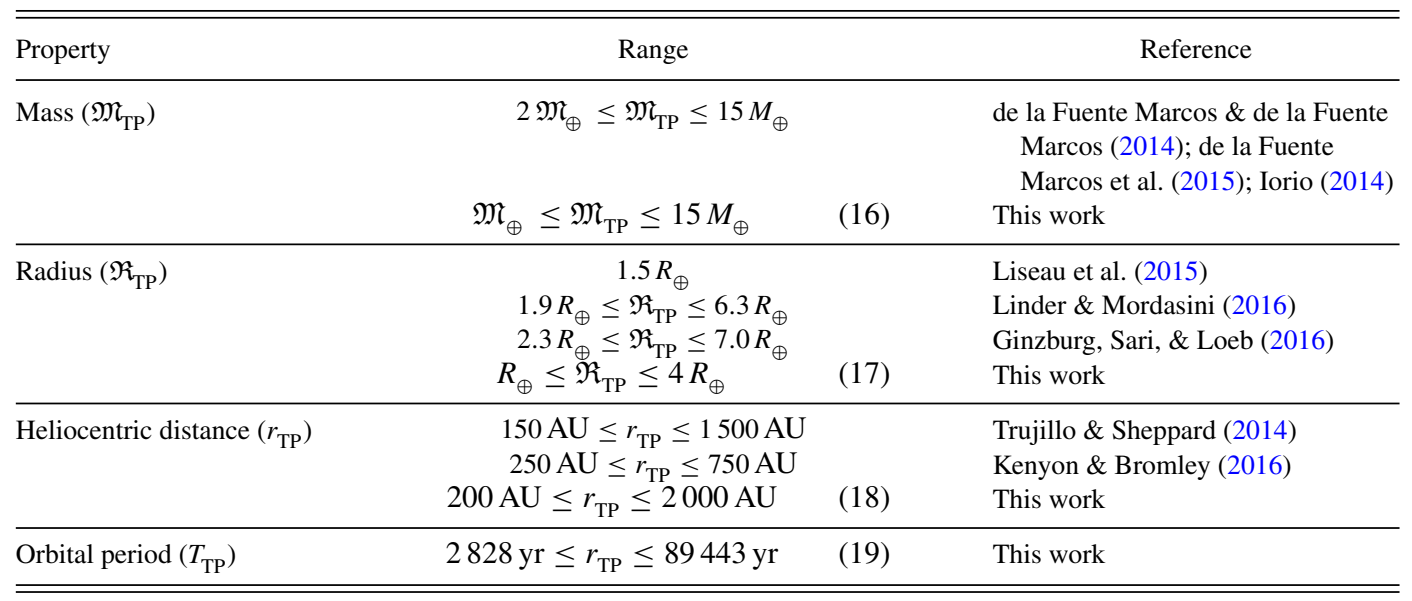

Fienga et al. (2016), Fortney et al. (2016), Ginzburg et al. (2016), Kenyon \& Bromley (2016), and Linder \& Mordasini (2016) to extend the ranges of mass (from one to fifteen Earth masses, $M_{\oplus}$ ), radius (from one to four Earth radii, $R_{\oplus}$ ) and available heliocentric distances (from 200 to $2000 \mathrm{AU}$ ) of any other trans-Plutonian planet. The orbital period of P9 has been taken as approximately equal to $18520 \mathrm{yr}$, following Batygin \& Brown (2016). In the case of a trans-Plutonian planet, other than $\mathrm{P} 9$, the range of possible values for the orbital period goes from 2828 to $89443 \mathrm{yr}$.

\section{QUANTITATIVE ANALYSIS OF THE FEASIBILITY OF THE DETECTION OF NEMESIS, TYCHE, AND TRANS-PLUTONIAN PLANETS USING GLM}

\subsection{Deflection angle in the gravitational field of Nemesis, Tyche, and a trans-Plutonian planet}

We consider the problem of the possibility of detecting either Nemesis, Tyche, or a trans-Plutonian planet that is playing the role of a gravitational lens using the following assumptions about the gravitational lens and the electromagnetic radiation coming from the background source.

1. A gravitational lens (Nemesis, Tyche, or a transPlutonian planet less massive than Neptune) is a spherical body of radius $\mathfrak{R}$ and mass $\mathfrak{M}$ with a spherically symmetric distribution of matter. Given its size and distance from the observer, the gravitational lens has a negligible angular dimension.

2. The gravitational influence of the major planets and other bodies of the Solar System on the light beams coming from the background source in the vicinity of the gravitational lens will be neglected.

3. Absorption of light by the interplanetary medium will also be neglected.
The electromagnetic radiation of the background star (source) can be considered as the photon beam. The dynamic photon mass $m_{\gamma}$ is determined using the Einstein's formula:

$$
m_{\gamma}=\frac{E_{\gamma}}{c^{2}}=\frac{p_{\gamma}}{c}
$$

where $p_{\gamma}$ is the photon momentum, $E_{\gamma}$ is the photon energy, and $c$ is the speed of light in a vacuum.

According to Newton's law of gravitation, the attraction force $\left(\boldsymbol{F}_{\mathrm{G}}\right)$ acting on a photon at the distance $r$ from the lens, directed along the line connecting both objects, is represented as:

$$
\boldsymbol{F}_{\mathrm{G}}=-\frac{G m_{\gamma} \mathfrak{M}}{r^{3}} \boldsymbol{r}
$$

where $G=6.674 \times 10^{-11} \mathrm{~m}^{3} \mathrm{~kg}^{-1} \mathrm{~s}^{-2}$ is the universal gravitational constant, $\mathbf{r}$ is the radius-vector of the photon, drawn from the centre of the lens, and $r$ is its module.

We assume that the original photon was moving along a straight line with the impact parameter $b$ (see Figure 2a). As a result of interaction with the lens, the photon will move along a geodesic curve, approaching the lens. The deflection angle $(\theta)$ of the photon $\gamma$ is the angle between the initial and final momenta of photons (see Figure $2 \mathrm{a}$ ). The deviation from the straight running depends on the body mass $\mathfrak{M}$ and the impact parameter $b$.

We consider the case of weak gravitational fields for a spherical body when the curvature of the trajectory of a photon can be neglected in the calculations; in other words, we assume that the photon moves in a straight line $A B$ (see Figure 2b) all the interaction time.

Using the law of change of momentum and the Cartesian coordinate system, which is shown in Figure 2b, we obtain the projections of the momentum change (relative to the 

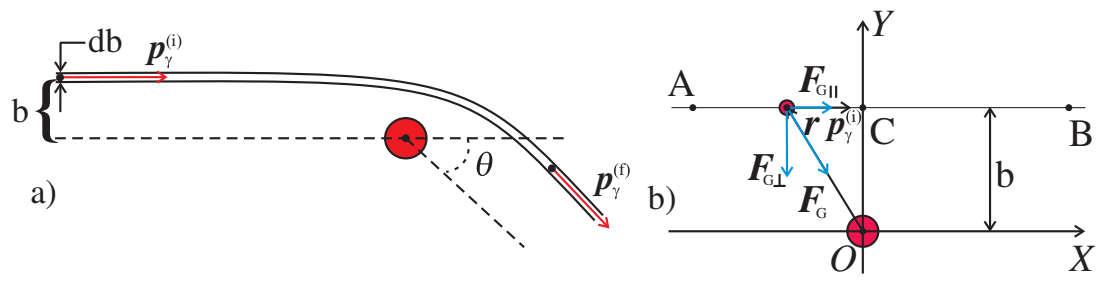

Figure 2. The scattering of a photon in the gravitational field of a lens.

initial direction of motion) in the form of

$$
\left.\begin{array}{l}
\Delta p_{\|}=-\frac{G m_{\gamma} \mathfrak{M}}{c} \int_{-\infty}^{+\infty} \frac{x d x}{\left(x^{2}+b^{2}\right)^{\frac{3}{2}}}, \\
\Delta p_{\perp}=-\frac{G m_{\gamma} \mathfrak{M} b}{c} \int_{-\infty}^{+\infty} \frac{d x}{\left(x^{2}+b^{2}\right)^{\frac{3}{2}}} .
\end{array}\right\}
$$

Obviously, the first integral of the system (22) is zero (Vygodsky 2006).

Performing a series of mathematical transformations, we obtain for the second integral the following result:

$$
\Delta p_{\perp}=-\frac{2 G m_{\gamma} \mathfrak{M}}{b c}=-\frac{2 G p_{\gamma}^{(\mathrm{i})} \mathfrak{M}}{b c^{2}}
$$

The result is obtained in the Newtonian approximationpotential theory of gravitation. However, Albert Einstein predicted that the exact gravitational theory must be tensor theory, and the curvature of space gives as large contribution to the final result in $\Delta p_{\perp}$ as Newton's theory of gravitation, according to Landau \& Lifshitz (2003). Hence, we write the following expression for the momentum change:

$$
\Delta p_{\perp}=-\frac{4 G p_{\gamma}^{(\mathrm{i})} \mathfrak{M}}{b c^{2}}, \tan \theta=\frac{\left|\Delta p_{\perp}\right|}{p_{\gamma}^{(\mathrm{i})}}=\frac{4 G \mathfrak{M}}{b c^{2}} .
$$

Since the angle $\theta$ is small, in practice it is customarily measured in arcsec:

$$
\theta^{\prime \prime}=\frac{4 G \mathfrak{M}}{b c^{2}} \times 206265^{\prime \prime}=\frac{2 \Re_{\mathrm{Sh}}}{b} \times 206265^{\prime \prime},
$$

where $\mathfrak{R}_{\mathrm{Sh}}$ is the Schwarzschild radius for a lens, which is defined by the expression:

$$
\mathfrak{R}_{\mathrm{Sh}}=\frac{2 G \mathfrak{M}}{c^{2}} .
$$

Using (1) and (6), we obtain the following ranges of possible values for $\mathfrak{R}_{\mathrm{Sh}}$ (in $\Re_{J}$ ) for Nemesis, Tyche, P9, and a trans-Plutonian planet, other than $\mathrm{P} 9$ :

$$
\left.\begin{array}{l}
5.120 \times 10^{-7} \leq \frac{\Re_{\mathrm{Sh}}^{N}}{R_{\mathrm{J}}} \leq 2.888 \times 10^{-6}, \\
3.939 \times 10^{-8} \leq \frac{\mathfrak{R}_{\mathrm{Sh}}^{T}}{R_{\mathrm{J}}} \leq 5.120 \times 10^{-7}, \\
1.389 \times 10^{-8} \leq \frac{\mathfrak{R}_{\mathrm{Sh}}^{P 9}}{R_{\oplus}} \leq 2.083 \times 10^{-8}, \\
1.389 \times 10^{-9} \leq \frac{\mathfrak{R}_{\mathrm{Sh}}^{T P}}{R_{\oplus}} \leq 2.083 \times 10^{-8} .
\end{array}\right\}
$$

The angle $\theta$ arcsec, according to (24), does not depend on photon frequency (wavelength). This property is called the achromatic effect of the lensing.

Figure $3 \mathrm{a}$ illustrates the deflection angle $\theta$ arcsec dependence on Nemesis' mass (within the range 13 to $73 M_{\mathrm{J}}$ ) for the impact parameter $b=1.5 \times R_{\mathrm{J}}$. This dependence is linear with the angle and varies within the range $0.14-0.80$ arcsec. In the case of Tyche (see Figure 4a), when the mass of the planet varies within the range of 1 to $13 M_{\mathrm{J}}$, the angle $\theta$ arcsec has the values $0.012-0.140$ arcsec. Figures $5 \mathrm{a}, 6 \mathrm{a}$ show the regions of possible values of the deflection angle $\theta$ arcsec as a function of the mass for P9 and a trans-Plutonian planet, other than P9, respectively. Any theoretical dependencies on $\mathfrak{M}_{P 9}\left(\mathfrak{R}_{P 9}\right), \mathfrak{M}_{T P}\left(\mathfrak{R}_{T P}\right)$, have not been taken into account because they are defined by the unknown internal structure of the hypothetical planet. It is obvious that the order of the deflection angle value is $\mathcal{O}$ (1 mas).

In Figures $3 b-6 b$, the curves of the deflection angle $\theta$ arcsec dependence on the impact parameter $b$ (expressed in $R_{\mathrm{J}}$ and $R_{\oplus}$ ) in the cases of Nemesis, Tyche, P9, and a trans-Plutonian planet, different from P9, are shown. There are three curves for the case of Nemesis corresponding to three different values of the mass $\mathfrak{M}_{\mathrm{N}}=\{13,45,73.3\} M_{\mathrm{J}}$ (low-mass brown dwarf, intermediate-mass brown dwarf, and massive brown dwarf, respectively). In the case of Tyche, there are four curves corresponding to values of the mass $\mathfrak{M}_{\mathrm{T}}=\{1,4,7,13\} M_{\mathrm{J}}$ (Jupiter-like planet, intermediate-mass jovian planet, massive jovian planet, very massive jovian planet). In the case of P9, three curves are shown. They correspond to three different values of the mass $\mathfrak{M}_{\mathrm{P} 9}=\{10,12.5,15\} M_{\oplus}$ (massive Super-Earth with $\mathfrak{R}_{\mathrm{P} 9}=2 R_{\oplus}$, mega-Earth or miniNeptune with $\Re_{\mathrm{P} 9}=3 R_{\oplus}$, and Neptune-like planet with $\left.\mathfrak{R}_{\mathrm{P} 9}=4 R_{\oplus}\right)$. In the case of a trans-Plutonian planet, other than P9, four curves are shown. They correspond to four different values of the mass $\mathfrak{M}_{\mathrm{TP}}=\{1,5,10,15\} M_{\oplus}$ (Earth-like planet with $\mathfrak{R}_{\mathrm{TP}}=R_{\oplus}$, intermediate-mass Super-Earth with $\mathfrak{R}_{\mathrm{TP}}=1.5 R_{\oplus}$, massive SuperEarth with $\Re_{\mathrm{TP}}=2 R_{\oplus}$, and Neptune-like planet with $\left.\mathfrak{R}_{\mathrm{TP}}=4 R_{\oplus}\right)$.

It is obvious that the deflection angle dependence on the impact parameter is hyperbolic. For all shown cases of Nemesis and Tyche, the value of the deflection angle is not less than 0.01 arcsec. This value is slightly smaller than the angular resolution of the HST ( $\beta \operatorname{arcsec}=0.05 \operatorname{arcsec}$ ), of the order of the angular resolution of the JWST (James Webb Space 

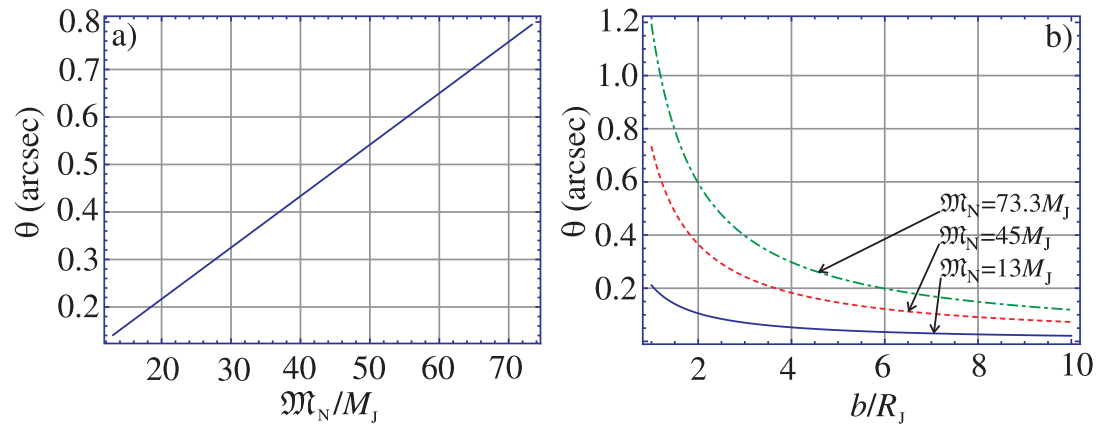

Figure 3. The curves of the deflection angle of the light beam in the gravitational field of Nemesis depending on (a) Nemesis' mass (expressed in Jupiter masses, $M_{\mathrm{J}}$ ), where $b=1.5 \times R_{\mathrm{J}}$; (b) the impact parameter $b$ (expressed in Jupiter radii, $R_{\mathrm{J}}$ ).
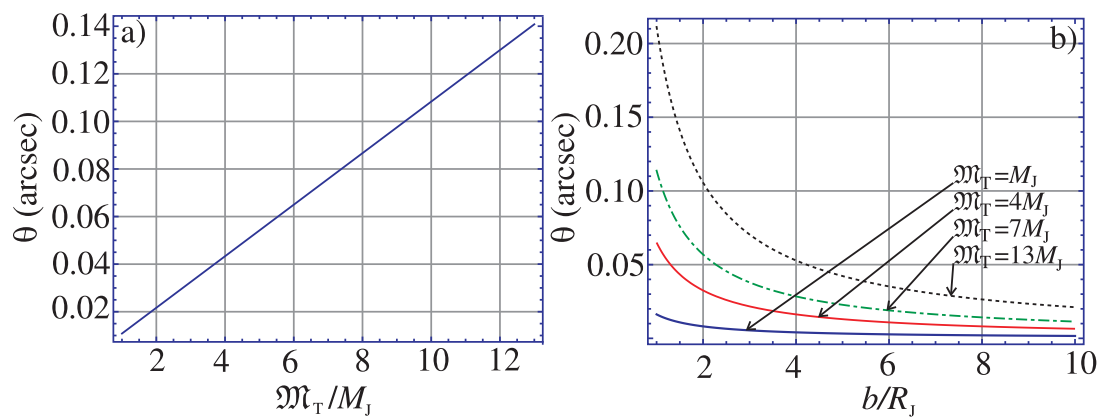

Figure 4. The curves of the deflection angle of the light beam in the gravitational field of Tyche depending on (a) Tyche's mass (expressed in Jupiter masses, $M_{\mathrm{J}}$ ), where $b=1.5 \times R_{\mathrm{J}}$; (b) the impact parameter $b$ (expressed in Jupiter radii, $R_{\mathrm{J}}$ ).
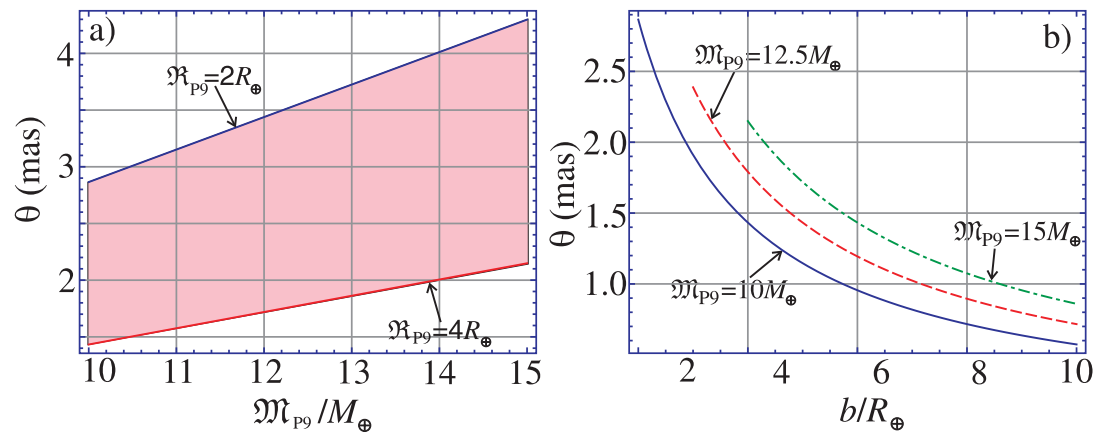

Figure 5. The curves of the deflection angle of the light beam in the gravitational field of Planet Nine depending on (a) its mass (expressed in Earth masses, $M_{\oplus}$ ), for two boundary values of its radius $\left(2 R_{\oplus}\right.$ and $\left.4 R_{\oplus}\right)$; (b) the impact parameter $b$ (expressed in Earth radii, $R_{\oplus}$ ).
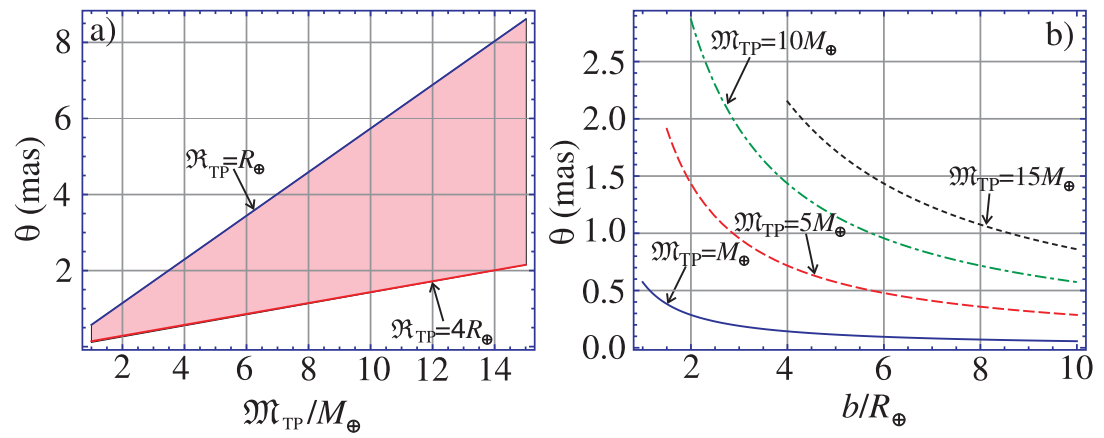

Figure 6. The curves of the deflection angle of the light beam in the gravitational field of transPlutonian planet depending on (a) its mass (expressed in Earth masses, $M_{\oplus}$ ), for two boundary values of its radius $\left(R_{\oplus}\right.$ and $4 R_{\oplus}$ ); (b) the impact parameter $b$ (expressed in Earth radii, $R_{\oplus}$ ). 
Telescope), that is $\beta$ arcsec $=0.018$ arcsec, and significantly larger than the theoretical estimate $(\beta \operatorname{arcsec}=0.003$ arc$\mathrm{sec}$ ) of the angular resolution of the future E-ELT (European Extremely Large Telescope) with diameter $D_{\mathrm{E}-\mathrm{ELT}}=39 \mathrm{~m}$ (Cunningham et al. 2008). Hence, $\theta$ arcsec will be measurable experimentally in the near future. The situation is different for $\mathrm{P} 9$ and any other trans-Plutonian planet. For all shown cases of $\mathrm{P} 9$ and a trans-Plutonian planet, the value of the deflection angle is not bigger than 2.9 mas. It is lower than the angular resolution of E-ELT. Hence, in the cases of $\mathrm{P} 9$ and a trans-Plutonian planet, other than $\mathrm{P} 9, \theta$ arcsec will not be measurable experimentally in the near future.

\subsection{Differential and total cross-sections of photon scattering in the gravitational field of the lens}

Since the trajectory of a single photon is not experimentally detected, what is usually measured is the flux of scattered particles $\left(F_{\text {sca }}\right)$. It is the number of particles scattered by a lens in a certain direction per unit time. The total cross section of scattering $\sigma$ is defined by

$$
\sigma=\frac{F_{\text {sca }}}{j_{0}}
$$

where $j_{0}$ is the flux density of particles (photons) that is the number of particles, moving in the direction of the target, passing through a unit area per unit time.

The elastic scattering process can be described by a differential cross-section of scattering which is equal to the ratio of the particle flux $\mathrm{d} F_{\text {sca }}$, scattered into the solid angle $\mathrm{d} \Omega$, to the flux density of incident particles $j_{0}$ and the solid angle $\mathrm{d} \Omega$ :

$$
\frac{\mathrm{d} \sigma}{\mathrm{d} \Omega}=\frac{\mathrm{d} F_{\text {sca }}}{j_{0} \mathrm{~d} \Omega}
$$

Using equations (28) and (23), we can express the differential cross-section of photon scattering as

$$
\left[\frac{\mathrm{d} \sigma}{\mathrm{d} \Omega}\right]=\left(\frac{4 G \mathfrak{M}}{c^{2}}\right)^{2} \frac{\cos \theta}{\sin ^{4} \theta} .
$$

The total cross-section of scattering is

$$
\begin{aligned}
\sigma_{\text {tot }} & =\int_{\Omega}\left[\frac{\mathrm{d} \sigma}{\mathrm{d} \Omega}\right] \mathrm{d} \Omega=2 \pi\left(\frac{4 G \mathfrak{M}}{c^{2}}\right)^{2} \int_{\theta_{\min }}^{\theta_{\max }} \frac{\cos \theta}{\sin ^{3} \theta} d \theta \\
& =4 \pi \Re_{\mathrm{Sh}}^{2}\left[\frac{1}{\sin ^{2} \theta_{\min }}-\frac{1}{\sin ^{2} \theta_{\max }}\right],
\end{aligned}
$$

where $\theta_{\max }$ is the maximum deflection angle, which can be achieved under these conditions. According to equation (23), the angle is maximal when the impact parameter $b$ is minimal. Obviously, it can not be less than the lens radius $\mathfrak{R}$, i.e., $b \geq \Re$, then

$$
\theta_{\max }=\arctan \left[\frac{4 G \mathfrak{M}}{\mathfrak{R} c^{2}}\right] \approx \frac{4 G \mathfrak{M}}{\mathfrak{R} c^{2}}=2 \frac{\mathfrak{R}_{\mathrm{Sh}}}{\mathfrak{R}} .
$$

$\theta_{\min }$ is the minimum deflection angle that can be detected by a telescope. Assuming that telescopic observations are performed in the visible part of the spectrum, this angle should be limited by the angular resolution of the telescope ( $\beta$ arcsec). As $\beta$ arcsec we will use the theoretical value of the resolution for the largest ground-based telescope, the E-ELT with a diameter $D_{\mathrm{E}-\mathrm{ELT}}=39 \mathrm{~m}$ :

$$
\begin{aligned}
& \theta_{\min }^{\prime \prime}=\beta^{\prime \prime}=\frac{120^{\prime \prime}}{D_{\mathrm{E}-\mathrm{ELT}}}=3.08 \times 10^{-3}{ }^{\prime \prime}, \Rightarrow \\
& \theta_{\min }=1.492 \times 10^{-8} .
\end{aligned}
$$

In the numerical analysis of the results, it is more convenient to use dimensionless quantities of the differential and the total cross-sections, which can be obtained from (29) and (30) dividing by the area of the geometric cross-section of the lens body $S_{\mathrm{G}}=\pi \mathfrak{R}^{2}$. The formulae for the differential cross-section and the total cross-section are

$$
\begin{aligned}
& S_{\Omega}=\frac{1}{S_{\mathrm{G}}}\left[\frac{\mathrm{d} \sigma}{\mathrm{d} \Omega}\right]=\frac{4}{\pi} x^{2} \frac{\cos \theta}{\sin ^{4} \theta}, \quad \theta_{\min } \leq \theta \leq \theta_{\max }, \\
& S_{\mathrm{tot}}=\frac{\sigma_{\mathrm{tot}}}{S_{\mathrm{G}}}=4 x^{2}\left[\theta_{\min }^{-2}-\left(\frac{1}{2 x}\right)^{2}\right], \quad x=\frac{\Re_{\mathrm{Sh}}}{\Re} .
\end{aligned}
$$

As for Nemesis and Tyche, the parameter $x$ belongs to the interval of possible values given by inequality (26).

Figures $7 \mathrm{a}$ and $8 \mathrm{a}$ illustrate the differential cross-section, $S_{\Omega}$, dependence on scattering of electromagnetic radiation in the gravitational field of Nemesis (Figure 7a), for three values of its mass, and Tyche (Figure 8a), for four different values of its mass, represented by the scattering angle $\theta$ arcsec (logarithmic scale). It can be seen that this relationship is linear; it is a function of the mass of the putative star or planet. When the mass of the gravitational lens decreases, the range of possible values for $x$ is narrowed.

In Figures $7 \mathrm{~b}$ and $8 \mathrm{~b}$, the curves of the total cross-section, $S_{\text {tot }}$, dependence on the value of the parameter $x$ for Nemesis and Tyche are shown (logarithmic scale). It is obvious that the desired relationship in this case corresponds to a continuous increase as $x$ grows. As it has been shown in the previous section, the value $\theta_{\max }$ is less than $\theta_{\min }$ for $\mathrm{P} 9$ and any other trans-Plutonian planet, then the values of the differential and total cross-sections, as given by equations (33) and (34), remain largely unaffected by changes in the scattering angle. Thus, the total cross-section is negative.

\subsection{Lensed images}

Let us consider the problem of imaging the primary point source of light by a gravitational lens. Suppose that a point gravitational lens (deflector) is located at the point $D$, a light source (background star) is located at $S$, and an observer is located at $O$ (see Figure 9). At large distances from the lens, the photons practically move along straight lines. Therefore, their trajectories can be approximated by two straight lines, broken near the body $D$ (at the points $A$ and $B$ ). The angle $\alpha$ 

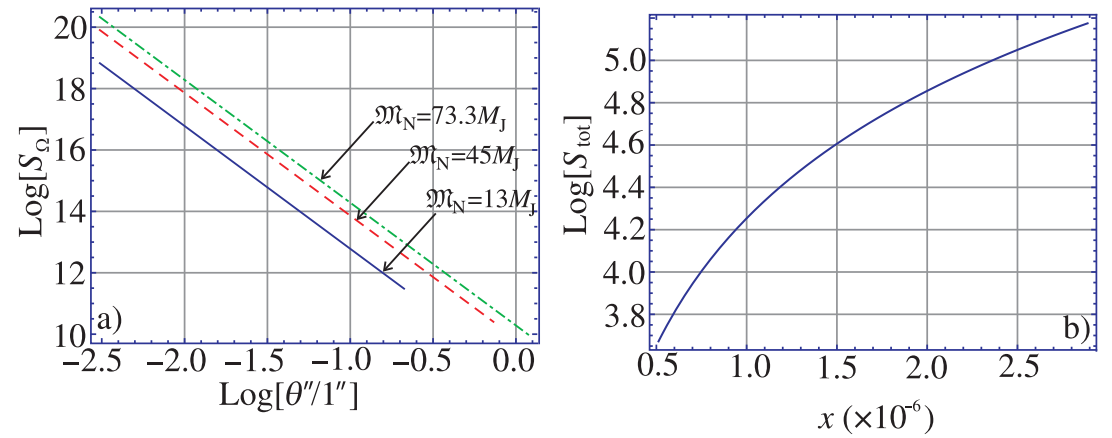

Figure 7. Dependence in the case of Nemesis of (a) the differential cross-section $S_{\Omega}$ of electromagnetic radiation scattering in the gravitational field of a lens (for three values of its mass) on the scattering angle $\theta \operatorname{arcsec}$, (b) total cross-section $S_{\text {tot }}$ dependence on the parameter $x$.
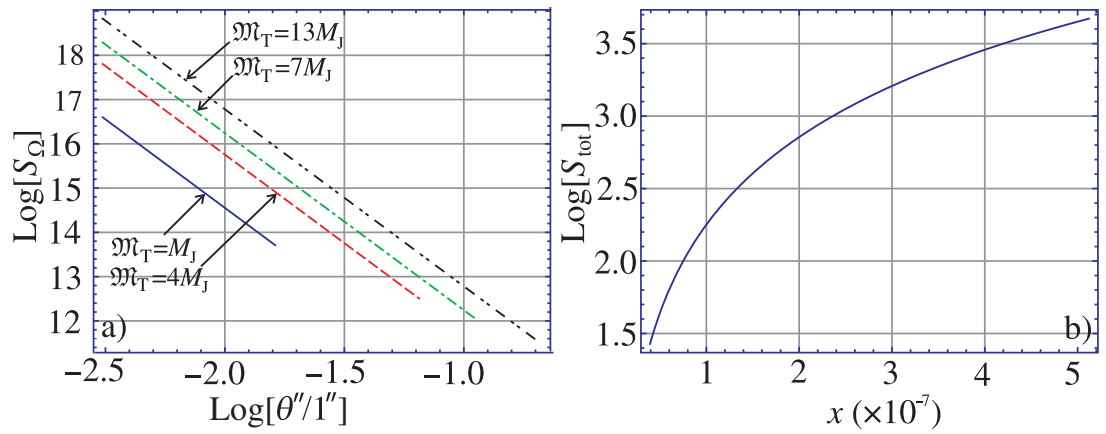

Figure 8. Dependence in the case of Tyche of (a) the differential cross-section $S_{\Omega}$ of electromagnetic radiation scattering in the gravitational field of a lens (for four values of its mass) on the scattering angle $\theta$ arcsec, (b) total cross-section $S_{\text {tot }}$ dependence on the parameter $x$.

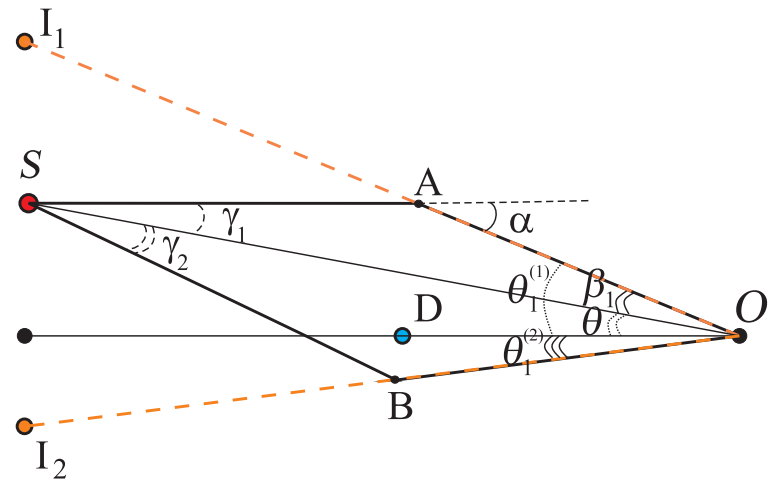

Figure 9. The curvature of the rays; the formation of the images of the source (S) in the gravitational field of a lens (D).

between them shows how the photon was bent, after passing through the gravitational field of the body $D$.

The angle between the direction of the deflector and the true position of the source $S$ is denoted by $\theta$. Two rays of light (shown by two bold lines), passed on the opposite side of the gravitating body, will be deflected from the initial direction towards the body. If the light source $S$ is far away from the body $D$, the rays converge at some distant point (Zakharov \& Sazhin 1998).
From Figure 9, with the simplest properties of a plane triangle for $\triangle A O S$, it is seen that

$$
\begin{aligned}
\alpha & =\beta_{1}+\gamma_{1}, \\
\theta_{1} & =\theta+\beta_{1},
\end{aligned}
$$

For the $\triangle A O S$, according to the law of sines, we have

$$
\frac{A O}{\sin \gamma_{1}}=\frac{S A}{\sin \beta_{1}} \text {. }
$$

Since the angles $\beta_{1}, \gamma_{1}$ are small, $\sin \beta_{1} \approx \beta_{1}, \sin \gamma_{1} \approx \gamma_{1}$. Let us also assume that the angle $\theta$ is small; so $S A \approx S D=$ $D_{\text {sd }}, A O \approx D O=D_{\mathrm{d}}$. As a consequence,

$$
D_{\mathrm{d}} \beta_{1}=D_{\mathrm{sd}} \gamma_{1} \text {. }
$$

From equalities (35), (36), taking into account that $\alpha=$ $4 G \mathfrak{M} /\left(b c^{2}\right)$, we have

$$
\theta_{1}=\theta+\alpha-\gamma_{1}=\theta+\frac{2 \Re_{\mathrm{Sh}}}{b}-\gamma_{1} .
$$

Then, from (37), we obtain $\gamma_{1}=\beta_{1}\left(D_{\mathrm{d}} / D_{\mathrm{sd}}\right)$. In the above expression, we take into account that $\beta_{1}=\theta_{1}-\theta$, according to (36). We also consider that the impact parameter is given by $b \approx D A \approx D_{\mathrm{d}} \times \theta_{1}$. As a result, we have

$$
\theta_{1}=\theta+\frac{2 \Re_{\mathrm{Sh}}}{D_{\mathrm{d}} \theta_{1}}-\left(\theta_{1}-\theta\right) \frac{D_{\mathrm{d}}}{D_{\mathrm{sd}}} .
$$




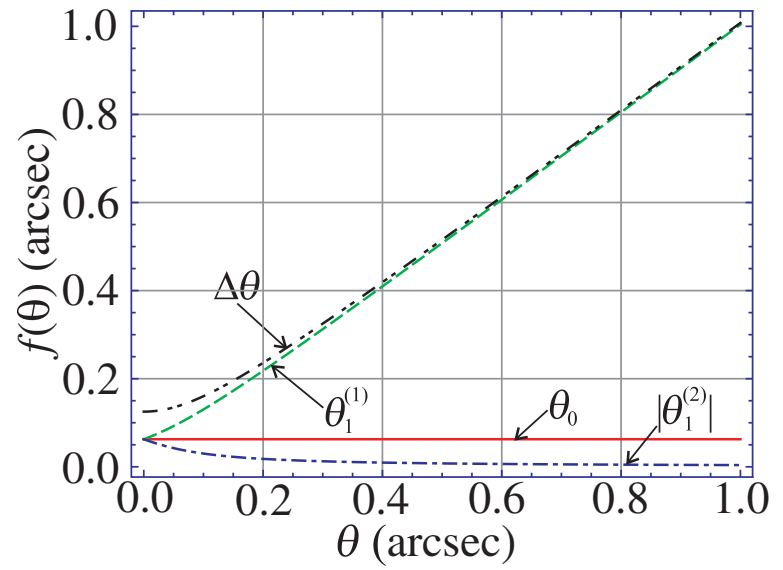

Figure 10. $\theta_{1}^{(1)},\left|\theta_{1}^{(2)}\right|, \Delta \theta=\theta_{1}^{(1)}-\theta_{1}^{(2)}, \theta_{0}$ dependencies on the deflection angle $\theta$, where $\mathfrak{M}_{N}=0.07 \mathfrak{M}_{\odot}, D_{\mathrm{d}}=30 \mathrm{kAU}$ (for a Jupiter-sized brown dwarf).

The equation can be reduced to the quadratic equation in the variable $\theta_{1}$ :

$$
\begin{gathered}
\theta_{1}^{2}-\theta \theta_{1}-\theta_{0}^{2}=0, \\
\theta_{0}^{2}=\left(\frac{2 \Re_{\mathrm{Sh}}}{D_{\mathrm{d}}}\right) \frac{D_{\mathrm{sd}}}{\left(D_{\mathrm{sd}}+D_{\mathrm{d}}\right)} .
\end{gathered}
$$

Where $\theta_{0}$ is the Einstein ring angular radius. The equation (38) is called the equation of the spherically symmetric point gravitational lens. It is conveniently expressed in terms of the source annual parallax $\left(\pi_{\mathrm{s}}\right)$ and the deflector annual parallax $\left(\pi_{\mathrm{d}}\right)$. Since $D_{\mathrm{sd}}=D_{\mathrm{s}}-D_{\mathrm{d}}$ and $D_{\mathrm{s}}=\frac{1 \mathrm{AU}}{\pi_{\mathrm{s}}^{\prime \prime}} 206265^{\prime \prime}$, $D_{d}=\frac{1 \mathrm{AU}}{\pi_{\mathrm{d}}^{\prime \prime}} 206265^{\prime \prime}$ (where $\pi_{\mathrm{s}}^{\prime \prime}, \pi_{\mathrm{d}}^{\prime \prime}$ are expressed in arcseconds), then

$$
\theta_{0}^{2}=\frac{2 \Re_{\mathrm{Sh} \odot} \pi_{\mathrm{d}}^{\prime \prime}}{206265^{\prime \prime} \times 1 \mathrm{AU}}\left(1-\frac{\pi_{\mathrm{s}}^{\prime \prime}}{\pi_{\mathrm{d}}^{\prime \prime}}\right)\left(\frac{\mathfrak{M}}{\mathrm{M}_{\odot}}\right),
$$

where $\Re_{\mathrm{Sh} \odot}=2 G \mathrm{M}_{\odot} c^{-2}=2.95 \mathrm{~km}$ is the Schwarzschild radius of the Sun. Expressing $\theta_{0}$ in arcseconds, we have

$$
\theta_{0}^{\prime \prime}=9.025^{\prime \prime} \times 10^{-2} \times \sqrt{\pi_{\mathrm{d}}^{\prime \prime}\left(1-\frac{\pi_{\mathrm{s}}^{\prime \prime}}{\pi_{\mathrm{d}}^{\prime \prime}}\right)\left(\frac{\mathfrak{M}}{\mathbf{M}_{\odot}}\right)} .
$$

For the background stars (belonging to the Milky Way), with annual parallax $\pi_{\mathrm{s}}^{\prime \prime} \sim 10^{-3}-10^{-2^{\prime \prime}}$, the above quantity (with $\pi_{\mathrm{s}}^{\prime \prime} \ll \pi_{\mathrm{d}}^{\prime \prime}$ ) can be expressed as

$$
\theta_{0}^{\prime \prime} \approx 9.025^{\prime \prime} \times 10^{-2} \times \sqrt{\pi_{\mathrm{d}}^{\prime \prime}\left(\frac{\mathfrak{M}}{\mathbf{M}_{\odot}}\right)} .
$$

It is clear that the equation (38) has two real roots corresponding to the two images of the source:

$$
\left.\begin{array}{l}
\theta_{1}^{(1)}=\frac{1}{2} \theta+\frac{1}{2} \sqrt{\theta^{2}+4 \theta_{0}^{2}} \\
\theta_{1}^{(2)}=\frac{1}{2} \theta-\frac{1}{2} \sqrt{\theta^{2}+4 \theta_{0}^{2}} \cdot
\end{array}\right\}
$$

Figure 10 shows the curves of both solutions of the equation (38), the value of $\Delta \theta=\theta_{1}^{(1)}-\theta_{1}^{(2)}$ related to the angle $\theta$, and the angular radius of the Einstein ring $\theta_{0}$. If the rapprochement of the source and the deflector is close $(\theta \rightarrow 0)$, then there is a bright pronounced phenomenon of lensing. In this case, the angular distance between the deflector and the images tends to be equal to the angular radius of the Einstein ring $\left(\theta_{0}\right)$ and the distance between the images tends to $2 \theta_{0}$. In fact, the images are moving on the Einstein ring.

Two images are not always visible. When the root $\theta_{1}^{(2)}$ becomes smaller than the angular radius of the deflector $\rho(\Re)$,

$$
\rho(\mathfrak{R})>\theta_{1}^{(2)}, \text { where } \rho(\mathfrak{R})=\frac{\mathfrak{R}}{D_{\mathrm{d}}},
$$

a ray of light eclipsed by the gravitational lens and the image $I_{2}$ becomes invisible to the observer. Hence, in this case, we can observe only one image which position does not coincide with the position of the source. Figures $11 \mathrm{a}, \mathrm{b}$ show the curves of $\theta_{0}^{(\min )}$ and $\theta_{0}^{(\max )}$ depending on heliocentric distance of the lens $D_{\mathrm{d}}$ for Nemesis and Tyche. It is clear that in the case of Nemesis its angular radius at any possible heliocentric distances is significantly smaller than the radius $\theta_{0}$. This means that the images, corresponding to Nemesis, are always visible and can be detected experimentally. In the case of Tyche, in most of the range of acceptable values $r_{\mathrm{T}}$, both images are visible only if the distance $r_{\mathrm{T}}>6060 \mathrm{AU}$ and Tyche's mass $\mathfrak{M}_{\mathrm{T}}>M_{\mathrm{J}}$. The situation for the cases of P9 and a trans-Plutonian planet, other than P9, is different. For descriptive reasons, we have defined the parameter $\Delta=$ $\rho-\theta_{0}$, as the difference of planet angular radius and Einstein ring angular radius.

For the entire ranges of distances and for all types of planets, $\Delta>0$ (see Figure $11 \mathrm{c}, \mathrm{d}$ ). Therefore, in principle, strong gravitational lensing is impossible because at least one image is always eclipsed by the body, but the gravitational microlensing is possible. Figure 12 illustrates the results of the modeling of trajectories of the source $S$ and the images $I_{1}$ and $I_{2}$ in the plane of the lens for two values of the impact parameter: (a) $\theta_{\mathrm{p}}=0.005 \operatorname{arcsec}$, (b) $\theta_{\mathrm{p}}=0.05$ arcsec. The arrows indicate the direction of their movement. It is important to note that the source $S$, the images, and the deflector are always located on the same straight line in the plane. One of the images $\left(I_{2}\right)$ separates from the lens and the other $\left(I_{1}\right)$ from the source when it is close to its Einstein ring.

\section{CONCLUSIONS}

In this paper, eight facts indirectly supporting the existence of a massive dark body at the Solar System periphery are reviewed. The basic physical properties and orbital characteristics of four different hypothetical candidates are considered. The solution of the problem of the deflection angle determined in the gravitational field of a spherical lens is given. The numerical analysis of the results obtained for the cases of Nemesis, Tyche, P9, and a trans-Plutonian planet, 

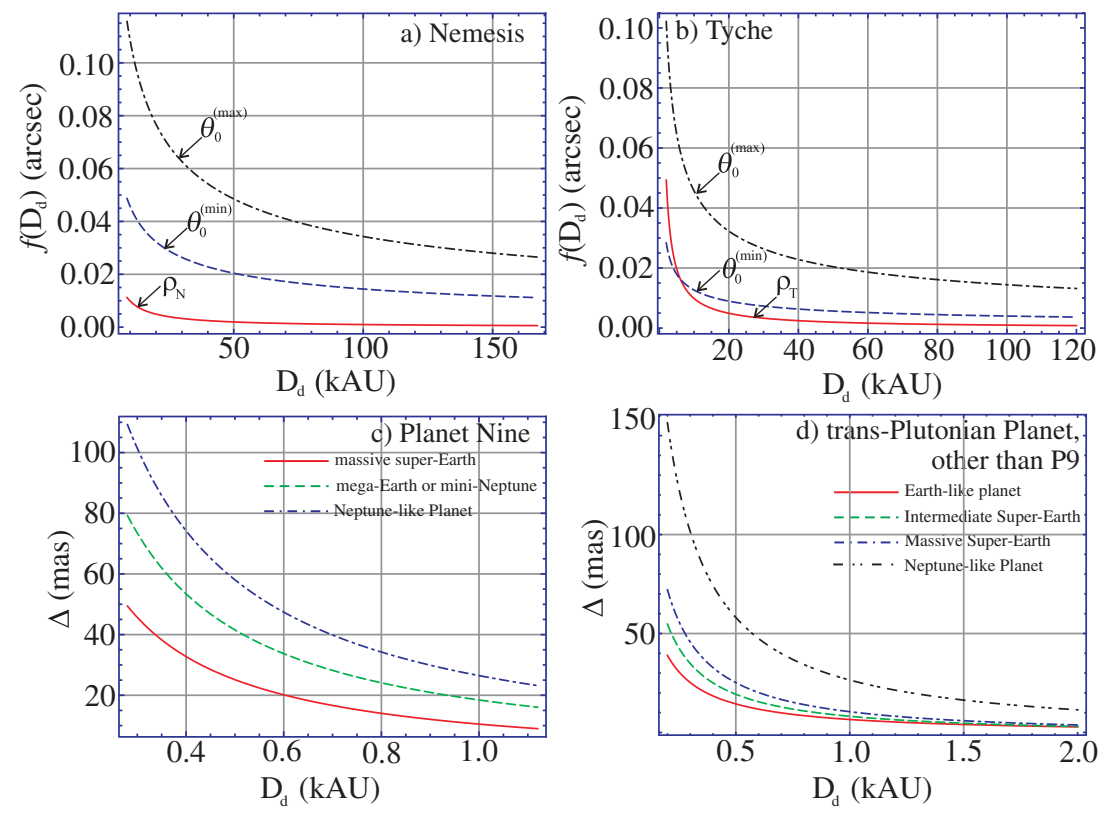

Figure 11. $\theta_{0}^{(\max )}=\theta_{0}\left(\mathfrak{M}_{\mathrm{N}, \mathrm{T}}^{(\max )}\right), \theta_{0}^{(\min )}=\theta_{0}\left(\mathfrak{M}_{\mathrm{N}, \mathrm{T}}^{(\min )}\right), \rho$ dependencies on heliocentric distance of the lens $D_{\mathrm{d}}$ in cases of Nemesis (a), Tyche (b). Dependence of $\Delta$ on heliocentric distance of the lens $D_{\mathrm{d}}$ for three types of planet in the case of Planet Nine (c) and for four types of planet in the case of a trans-Plutonian planet, other than Planet Nine (d), defined in subsection 4.1.
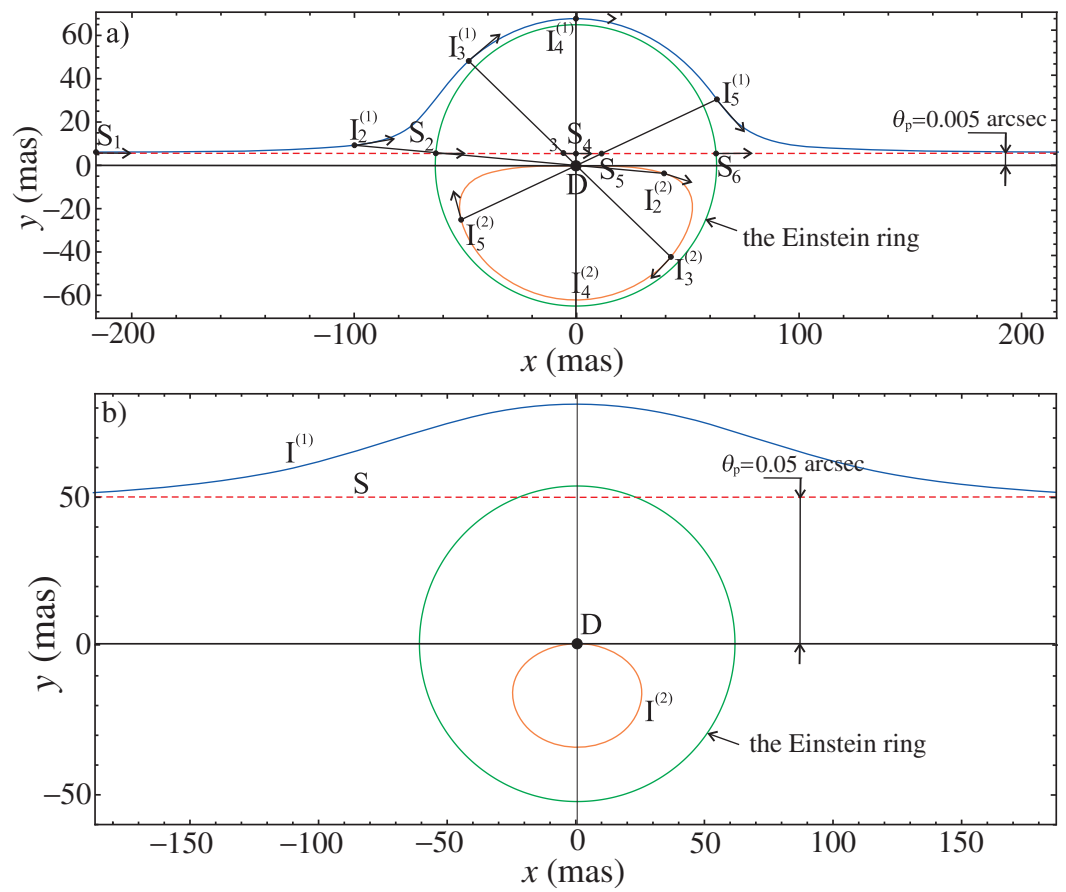

Figure 12. The trajectories of the source $(S)$ and its images $\left(I_{1}\right.$ and $\left.I_{2}\right)$, which were created by a gravitational lens (Nemesis with mass of $\mathfrak{M}_{N}=0.07 \mathfrak{M}_{\odot}$ and a corresponding distance of $\left.D_{\mathrm{d}}=30 \mathrm{kAU}\right)$, in the cases of $\theta_{\mathrm{p}}=0.005 \operatorname{arcsec}(\mathrm{a}), \theta_{\mathrm{p}}=0.05 \operatorname{arcsec}(\mathrm{b})$. The directions of motion of the source and its images as indicated by the arrows.

other than P9, are presented. It is shown that only in the cases of Nemesis and Tyche the value of the deflection angle will be measurable in the near future with the aid of the JWST and the E-ELT. Analytical results for the differential and total cross-sections of photon scattering in a weak grav- itational field corresponding to a spherical lens are included in its dimensionless form. It is shown that the corresponding function of dimensionless differential cross section $S_{\Omega}$ takes an extremely large value due to the very small deflection angles $\theta$ arcsec and a dependence of the type $\sin ^{-4} \theta$. Given 
the problem of the formation of images by a gravitational lens, it is shown that, in general, for the cases of Nemesis and Tyche, two images can be detected experimentally. Only in the case of Tyche with a mass close to the mass of Jupiter at a distance of less than 6060 AU from the Sun, Tyche could be resolved in one of the images. In the cases of P9 and any other trans-Plutonian planet, for the entire ranges of distances and for all types of planet strong gravitational lensing is impossible, in principle, but gravitational microlensing is possible. Photometry of gravitational lensing for the cases of Nemesis and Tyche and microlensing for the cases of P9 and a trans-Plutonian planet, other than $\mathrm{P} 9$, requires additional analyses out of the scope of the present work and will be covered in a forthcoming paper.

\section{ACKNOWLEDGEMENTS}

We thank the anonymous reviewers for careful reading of the paper. We appreciate all suggestions that significantly improved the manuscript.

\section{REFERENCES}

Batygin, K., \& Brown, M. E. 2016, AJ, 151, 22

Bhalerao, V., \& Vahia, M. N. 2005, BASI, 33, 1

Bickerton, S. J., Kavelaars, J. J., \& Welch, D. L. 2008, AJ, 135, 1039

Brown, M. E. 2004, BAAS, 36, 1553

Brown, M. E., \& Batygin, K. 2016, ApJL, 824, L23

Burrows, A., Hubbard, W. B., Saumon, D., \& Lunine, J. I. 1993, ApJ, 406, 1, 158

Chwolson, O. 1924, AN, 221, 329

Cooray, A. 2002, arXiv:0209.545

Cunningham, C., Evans, C., Monnet, G., \& Le Louarn, M. 2008, in SPIE Proceedings, Vol. 6986, ed. T. E. Andersen (Bellingham: SPIE Publications) 69860K

Davis, M., Hut, P., \& Muller, R. A. 1984, Nature, 308, 5961, 715

de la Fuente Marcos, C., \& de la Fuente Marcos, R. 2014, MNRASL, 443, 1, L59

de la Fuente Marcos, C., de la Fuente Marcos, R., \& Aarseth, S. J. 2015, MNRAS, 446, 2, 1867

Duquennoy, A., \& Mayor, M. 1991, A\&A, 248, 485

Einstein, A. 1936, Science, 84, 2188, 506

Fienga, A., Manche, H., Laskar, J., \& Gastineau, M. 2008, A \& A, 477,315
Fienga, A., Laskar, J., Manche, H., \& Gastineau, M. 2016, A \& A, 587, L8

Fienga, A., et al. 2011, CeMDA, 111, 363

Fienga, A., et al. 2009, A\&A, 507, 1675

Fortney, J. J., et al. 2016, ApJL, 824, L25

Gaudi, B. S., \& Bloom, J. S. 2005, ApJ, 635, 711

Gaudi, B. S., \& Sackett, P. D. 2000, ApJ, 529, 56

Ginzburg, S., Sari, R., \& Loeb, A. 2016, ApJ, 822, 1, L11

Gomes, R. S., Matese, J. J., \& Lissauer, J. J. 2006, Icarus, 184, 2 , 589

Heyl, J. 2010, MNRASL, 402, 1, L39

Iorio, L. 2014, MNRASL, 444, L78

Kalas, P., Graham, J. R., Clampin, M. C., \& Fitzgerald, M. P. 2006, ApJL, 637, 1, 57

Kenyon, S. J., \& Bromley, B. C. 2015, ApJ, 806, 42

Kenyon, S. J., \& Bromley, B. C. 2016, ApJ, 825, 33

Kononovich, E. V., \& Moroz, V. I. 2004, A Course of Introduction to Astronomy (2nd edn.; Moscow: Editorial URSS)

Landau, L. D., \& Lifshitz, E. M. 2003, Theoretical Physics. Field Theory (V2) (5th edn.; Moscow: FIZ-MATLIT)

Linder, E. F., \& Mordasini, C. 2016, A \& A, 589, A134

Liseau, R., et al. 2015, arXiv:1512.02652

Luhman, K. L. 2014, ApJ, 781, 4

Marois, C., et al. 2008, Science, 322, 1348

Matese, J. J., \& Whitmire, D. P. 1986, Icarus, 65, 37

Matese, J. J., Whitmire, D. P., \& Lissauer, J. J. 2006, EMP, 97, 3-4, 459

Matese, J. J., Whitmire, D. P., \& Whitman, P. G. 2011, Icarus, 2, 926

Muller, R. A. 2002, GSASP, 356, 659

Paczynski, B. 1986a, ApJ, 301, 503

Paczynski, B. 1986b, ApJ, 304, 1

Raup, D. M., \& Sepkoski, J. J. 1984, PNAS, 81, 3, 801

Roques, F., Moncuquet, M., \& Sicardy, B. 1987, AJ, 93, 1549

Trujillo, C. A., \& Sheppard, S. S. 2014, Nature, 507, 471

Vlemmings, W. H. T., Ramstedt, S., Maercker, M., \& Davidsson, B. 2015, arXiv:1512.02650

Vygodsky, M. Y. 2006, Reference Book on the Higher Mathematics (5th edn.; Moscow: AST, Astrel)

Whitmire, D. P. 2016, MNRASL, 455, 1, L114

Whitmire, D. P., \& Jackson, A. A. 1984, Nature, 308, 5961, 713

Yarris, L. 1987, Science Beat, Spring LBL Research Review (http:// www2.lbl.gov/Science-Articles/Archive/extinctions-nemesis. html)

Zakharov, A. F., \& Sazhin, M. V. 1998, UFN, 168, 10, 1041

Zhdanov, V. I., \& Gorpinchenko, D. V. 2012, UkJP, 57, 10, 1083

Zhu, W., et al. 2015, ApJ, 814, 2, 129 